\title{
Increased cortical hyperexcitability and exaggerated myoclonus with aging in benign adult familial myoclonus epilepsy.
}

\section{$\operatorname{AUTHOR}(\mathrm{S}):$}

Hitomi, Takefumi; Ikeda, Akio; Kondo, Takayuki; Imamura, Hisaji; Inouchi, Morito; Matsumoto, Riki; Terada, Kiyohito; ... Nagamine, Takashi; Shibasaki, Hiroshi; Takahashi, Ryosuke

\section{CITATION:}

Hitomi, Takefumi ...[et al]. Increased cortical hyperexcitability and exaggerated myoclonus with aging in benign adult familial myoclonus epilepsy.. Movement disorders 2011, 26(8): 1509-1514

\section{ISSUE DATE:}

2011-04-19

\section{URL:}

http://hdl.handle.net/2433/197307

\section{RIGHT:}

This is the peer reviewed version of the following article: Hitomi, T., Ikeda, A., Kondo, T., Imamura, H., Inouchi, M., Matsumoto, R., Terada, K., Kanda, M., Matsuhashi, M., Nagamine, T., Shibasaki, H. and Takahashi, R. (2011), Increased cortical hyperexcitability and exaggerated myoclonus with aging in benign adult familial myoclonus epilepsy. Mov. Disord., 26: 1509-1514, which has been published in final form at http://dx.doi.org/10.1002/mds.23653; この論文は出版 社版でありません。引用の際には出版社版をご確認ご利用ください。; This is not the published version. Please cite only the published version. 
Title: Increased cortical hyperexcitability and exaggerated myoclonus with aging in benign familial myoclonus epilepsy

Running title: Progression with aging in BAFME

Authors: Takefumi Hitomi MD $\mathrm{PhD}^{1 *}$, Akio Ikeda $\mathrm{MD} \mathrm{PhD}^{1}$, Takayuki Kondo $\mathrm{MD}^{1}$, Hisaji Imamura $\mathrm{MD}^{1}$, Morito Inouchi $\mathrm{MD}^{1}$, Riki Matsumoto $\mathrm{MD} \mathrm{Ph}^{1}$, Kiyohito Terada MD $\mathrm{PhD}^{2}$, Masutaro Kanda $\mathrm{MD} \mathrm{PhD}^{3}$, Masao Matsuhashi MD PhD ${ }^{4}$, Takashi Nagamine MD $\mathrm{PhD}^{4}$, Hiroshi Shibasaki MD $\mathrm{PhD}^{3}$, Ryosuke Takahashi MD $\mathrm{PhD}^{1}$

1: Department of Neurology, Kyoto University School of medicine

2: Department of Epilepsy, National Epilepsy Center, Shizuoka Institute of Epilepsy and Neurological Disorders

3: Department of Neurology, Takeda General Hospital

4: Human Brain Research Center, Kyoto University Graduate School of Medicine

${ }^{*}$ Current address: Department of Respiratory Care and Sleep Control Medicine, Kyoto University School of medicine

\section{Address for Correspondence}

Akio Ikeda MD, PhD

Department of Neurology, Kyoto University Hospital

54 Shogoin-Kawaharacho, Sakyo-ku, Kyoto 606-8507, Japan

Tel. (+81)-75-751-3772, Fax. (+81)-75-751-9416, e-mail; akio@kuhp.kyoto-u.ac.jp

The number of references $=24$, the number of figures $=3$, the number of tables $=2$

Word count: title $=99$ character, abstract $=241$ words, text $=2569$ words 


\title{
Financial Disclosure
}

This study was supported by a Research Grant (22-3) for Nervous and Mental

Disorders from the Ministry of Health and Welfare, the Grant-in-Aid for Scientific Research (C2) 18590935 from the Japan Ministry of Education, Culture, Sports, Science and Technology (MEXT), and a Research Grant from the Japan Epilepsy Research Foundation.

\section{Conflict of interest}

The authors report no conflict of interest.

We confirm that we have read the Journal's position on issues involved in ethical publication and affirm that this report is consistent with those guidelines.

\section{Key words}

benign adult familial myoclonus epilepsy (BAFME),

giant somatosensory evoked potential (giant SEP),

cortical myoclonic tremor, aging

\author{
Abbreviations \\ benign adult familial myoclonus epilepsy (BAFME), \\ somatosensory evoked potential (SEP), \\ jerk-locked back averaging (JLA)
}




\section{Abstract}

Background: The clinical implications of enlarged early cortical components of somatosensory evoked potentials (giant SEPs) in benign adult familial myoclonus epilepsy (BAFME) remains unknown.

Methods: SEPs following electrical stimulation of the median nerve at the wrist were studied in 16 patients with a clinical diagnosis of BAFME (7 men and 9 women, mean age: $51 \pm 18$ years) and 19 age-matched apparently healthy control subjects (11 men and 8 women, mean age $49 \pm 18$ years).

Results: Giant SEPs were observed in 13 of the 16 patients. P25 and N35 amplitudes in the patient group were $11.4 \pm 6.1 \mu \mathrm{V}$ and $19.2 \pm 11.5 \mu \mathrm{V}$, respectively, and both were significantly larger compared with those in control subjects $(P=0.008$ for P25 and $P<0.0001$ for N35). There was a significant positive relationship between age at the SEP examination and N20, P25 and N35 amplitudes both in the patient and control groups $(P<0.05)$. The linear regression gradient of the N35 amplitude with respect to age was significantly larger in the patient group than in the control group ( $P$ $=0.04)$. Furthermore, regression analysis showed a significant positive relationship between the myoclonus rating scale and age at the time of the SEP examination $(R=$ 0.645, $P=0.007)$.

Conclusions: SEP amplitude increased with age in patients with BAFME to a greater extent than in the control subjects, which suggests a progressive increase in cortical excitability based on a progressive pathophysiology in BAFME. 


\section{Introduction}

Benign adult familial myoclonus epilepsy (BAFME) is an autosomal dominant disease characterized by tremulous myoclonus resembling essential tremor and infrequent generalized seizures. BAFME is not currently listed by the International League Against Epilepsy, and previous reports cited BAFME as a probable epileptic syndrome within the idiopathic generalized epilepsies [1]. The term 'benign' was assigned because of the non-progressive course and rare occurrence of generalized convulsions associated with this condition. BAFME has been reported from Japan and Europe with various names, including cortical tremor [2], BAFME [3], familial cortical myoclonic tremor [4], familial cortical tremor with epilepsy [5], familial cortical myoclonic tremor with epilepsy [6] and familial adult myoclonic epilepsy [7]. At least as far as the cases reported from Japan are concerned, these diverse terms can be suggested to represent the same clinical entity. Electrophysiological studies in patients with BAFME disclosed the features of cortical reflex myoclonus including excessively enlarged cortical components of somatosensory evoked potentials (giant SEPs), an enhanced long loop reflex (C-reflex) and the occurrence of cortical spikes preceding myoclonic jerks demonstrated by jerk-locked back averaging (JLA) [2, 4]. Giant SEPs are considered to represent cortical hyperexcitability of the primary somatosensory cortex in response to somatosensory stimuli [8] and to result from pathological enhancement of normal SEP components $[9,10]$. However, the relationship between the clinical profiles of patients and giant SEPs in BAFME remains undetermined.

In the present study, we aimed at elucidating how giant SEPs are related to clinical profiles of patients with BAFME. Since SEPs become larger with aging in normal subjects [11], we paid special attention to the effect of aging on giant SEPs. If 
the giant SEPs also were shown to be larger in aged patients with BAFME, it would be worthwhile to clarify whether the enlargement is proportional to the physiological enlargement found with normal aging or is related to progressive changes in the underlying pathophysiology. 
Hitomi et al 6

\section{Subjects and methods}

\section{Subjects}

Diagnostic criteria for BAFME used in this study were: (a) tremulous myoclonus and infrequent generalized seizures appearing after the second decade of life, (b) autosomal dominant inheritance, (c) lack of cognitive decline and other neurological symptoms, (d) features of cortical reflex myoclonus demonstrated by electrophysiological studies, and (e) lack of clear progression apparent through clinical observation (i.e., no severe disability in daily life such as walking, feeding, speech and intellectual function, except for writing due to cortical tremor). However, some patients with either tremulous myoclonus or infrequent generalized seizures alone were also included in this study, if (1) at least one electrophysiological indication of cortical reflex myoclonus (giant SEPs, C-reflex, or cortical spike preceding myoclonic jerks by JLA) was demonstrated and if (2) at least one family member had the diagnosis of BAFME, suggestive of autosomal dominant inheritance.

Sixteen patients (7 men and 9 women) with the clinical diagnosis of BAFME who were under care in 3 different hospitals during the last 20 years were studied with regard to SEPs. The mean age at the time of SEP recording was $51 \pm 18$ years (S.D., range 27-76 years) (Table 1). Six of these patients (No. 3, 4, 7, 8, 10 and 11) were previously reported for different purposes [2, 4]. Among the 16 BAFME patients, 14 had tremulous myoclonus and 11 had infrequent generalized seizures (Table 1). Two patients (Patients 6 and 16) had only equivocal (Patient 6) or transient (Patient 16) fine hand tremor. The onset age of tremulous myoclonus was $38 \pm 16(10-70)$ years and that of generalized seizure was $41 \pm 19$ (17-73) years. Generalized seizure occurred after the onset of the tremulous myoclonus except in 1 patient (Patient 10). The duration of tremulous myoclonus was $15 \pm 13$ (2-44) years. The severity of 
tremulous myoclonus was classified according to the authors' previous report as shown in the supporting material (available online) and Table 1 [12]. Scoring of the severity of tremulous myoclonus was retrospectively done by one of the authors (A.I.) based on detailed descriptions of neurological findings in patients' medical charts.

SEPs were recorded in 19 age-matched apparently healthy control subjects (11 men and 8 women, mean age $49 \pm 18$ years, range 22-74 years) at Kyoto University Hospital. The institutional review board of Kyoto University Hospital approved performance of SEP recording in control subjects (No. E678). In the BAFME patients, SEP studies were done as a part of their clinical evaluation in each hospital. Informed consent for participation in the study was obtained from all subjects.

\section{Methods}

Scalp SEPs in response to stimulation of the median nerve at the wrist were examined in all patients and control subjects. Details of recording conditions are given in the supporting material (available online). Nomenclature of the peaks (N20, P25 and N35) was adopted from the authors' previous report [10]. Amplitude of P25 and N35 was measured from the preceding peak of opposite polarity [10, 11, 13], and that of N20 was measured from the pre-stimulus baseline. Since the SEP amplitude was shown to increase with age in control subjects $[11,14]$, the upper limit of the normal range of the amplitude was set at the mean +3 S.D. of values obtained from the 10 control subjects in the younger subgroup ( $\leq 50$ years old) and 9 control subjects in the older subgroup ( $>50$ years): P25 $>10.0 \mu \mathrm{V}$ or $\mathrm{N} 35>8.1 \mu \mathrm{V}$ for the younger subgroup and $\mathrm{P} 25>20.0 \mu \mathrm{V}$ or $\mathrm{N} 35>14.8 \mu \mathrm{V}$ for the older subgroup. 


\section{Statistical Analysis}

In order to evaluate the effect of aging on myoclonus, we investigated the relationship between the myoclonus rating scale and age at the SEP examination by simple linear regression analysis.

To analyze SEP data, we first compared the latencies and amplitudes of the early cortical components (N20, P25 and N35) between electrodes C3/C4 and C3'/C4' in the patient group. Since there was no significant difference either in latency or amplitude between these two electrode pairs, the Mann-Whitney U test was used to compare the latencies and amplitudes of those 3 peaks in the patient group with data obtained from C3'/C4' in the control group. In the patient group, we investigated the relationship between clinical profiles (age at the time of SEP examination, age at onset, duration of tremulous myoclonus, and the myoclonus rating scale) and SEP parameters by simple linear regression analysis. SEP parameters were also analyzed with respect to other clinical factors (gender, presence of seizure, and type of anticonvulsant used) by the Mann-Whitney U test. In the control group, simple linear regression was used to evaluate the relationship between age at the time of the SEP examination and SEP parameters. If there was a significant positive relationship between age at the time of the SEP examination and SEP parameters either in the patient group or in the control group, the regression gradient between age and SEP parameters in the patient group was compared with that in the control group. The level of statistical significance was set at $P<0.05$. 
Hitomi et al 9

\section{Results}

\section{Myoclonus rating scale according to age in patients with BAFME}

Myoclonus was marked and severe in 4 of the aged patients (Patients 3, 4, 11 and 13), mild in 3 patients (Patients 1, 2, and 14), moderately severe in 7 patients, and absent in the remaining 2 patients (Patients 6 and 16) (Table 1). Regression analysis showed a significant positive relationship between the myoclonus rating scale and age at the time of the SEP examination $(R=0.645, P=0.007)$ (Fig. 1$)$.

\section{Scalp SEPs}

In patients with BAFME, there was no significant difference in latencies or amplitudes of SEPs between C3/C4 and C3'/C4'. Therefore, we analyzed the SEP data on all patients as a "single" group.

Latencies of SEPs in the patient group were not significantly different from those in the control group (Table 2). With regard to amplitudes, P25 and N35 were significantly larger in the patient group than in the control group $(P=0.008$ for $\mathrm{P} 25$ and $P<0.0001$ for N35) (Table 2). In contrast, the N20 amplitude was smaller in the patient group than in the control group $(P=0.006)$.

According to the definition of giant SEPs adopted in this study, 13 patients had giant SEPs (Table 1). In contrast, an enlarged P25 or N35 was not noted in 3 patients (Patients 2, 4 and 8) although they all 3 had at least one of the electrophysiological features of cortical reflex myoclonus. 


\section{Relationship between clinical profiles and SEP parameters}

(a) Patient group

As for latencies, there was no significant relationship between clinical profiles and early cortical components of SEPs (N20, P25 and N35) in BAFME patients. With regard to amplitudes, regression analysis showed a significant positive relationship between age at the time of the SEP examination and N20 $(R=0.675, P=$ 0.0057), P25 ( $R=0.689, P=0.0032)$ and N35 $(R=0.605, P=0.013)$ (Figs. 2A, 2B, and 2C). Representative cases from the same pedigree are shown in Fig. 3. A 58-year-old man (Patient 1) had giant SEPs (Fig. 3A), while his 34-year-old son (Patient 2) did not (Fig. 3B). A similar tendency was observed in the other pedigrees (Patients 10 and 11, and Patients 14 and 15). Otherwise, there was no significant relationship between clinical profiles (onset age, disease duration of tremulous myoclonus and the myoclonus rating scale) and SEP amplitudes. There was no significant difference in the SEP amplitudes with regard to gender, experience of seizure or administration of anticonvulsants.

(b) Control group

As for the control group, there was also a significant positive relationship between age at the time of the SEP examination and N20 $(R=0.49, P=0.0331)$, P25 $(R=0.732, P=0.0004)$ and N35 $(R=0.559, P=0.0004)$ amplitudes measured at C3'/C4' (Figs. 2A, 2B, and 2C).

(c) Comparison between patient and control groups

Comparison of the regression gradient for age and SEP amplitudes between the two groups showed no significant difference in terms of N20 and P25 amplitudes. 
The regression for N20 amplitude in the patient group $(\mathrm{Y}=0.468+0.047 \times \mathrm{X})$ was almost the same as that in the control group $(\mathrm{Y}=1.967+0.048 \times \mathrm{X})($ Fig. $2 \mathrm{~A})$. The regression for P25 amplitude in the patient group $(\mathrm{Y}=-0.308+0.233 \times \mathrm{X})$ was almost twice as large as that in the control group $(\mathrm{Y}=-0.156+0.139 \times \mathrm{X})$, but the difference did not reach statistical significance (Fig. 2B). In contrast, the regression gradient of N35 amplitude was significantly higher in the patient group ( $\mathrm{Y}=-0.43$ $+0.389 \times \mathrm{X})$ than in the control group $(\mathrm{Y}=-0.702+0.089 \times \mathrm{X})(P=0.04)($ Fig. 2C). 


\section{Discussion}

With regard to the diagnostic criteria of BAFME, some patients with myoclonus alone [15] and others with generalized seizures alone were also included in previous studies of BAFME $[16,17]$. These criteria are similar to those in the present study. The lack of a seizure history in 2 young patients in the present series (Patients 8 and 9) may be due to the rare incidence of seizures in this condition as described previously [7]. In genetic investigations of BAFME, the condition in Japanese families was linked to chromosome 8q [7, 18] and in European families was linked to chromosomes 2p [16, 17, 19] and 5p [20]. However, another report showed no linkage to chromosome 8q in some Japanese families [21]. Moreover, a possible anticipation was suggested in a Japanese pedigree [22]. Overall, the genetic diagnosis of BAFME has not been established and remains unclear. At present, clinical and electrophysiological findings and a positive, consistent family history are essential for the diagnosis of BAFME. Therefore, our inclusion criteria in the present series are considered to be appropriate. Discussion of the relationship between anticonvulsants and SEP amplitude is shown in the supporting material (available online).

The present study demonstrated that the early cortical components of SEPs, i.e., P25 and N35, were significantly enlarged in most patients with BAFME. Furthermore, both in the patient group and age-matched control group, all 3 peaks (N20, P25, and N35) were significantly larger in the aged than in the young subjects. Here, it is noteworthy that, among these 3 peaks, only N35, but not the N20 or P25, was found to become significantly larger with aging in the patient group than in the control group. The physiological mechanisms underlying this discrepancy remain to be elucidated, but may be closely related to the mechanisms of myoclonus generation. Therefore, it is conceivable that there is an excessive increase in cortical 
hyperexcitability with aging in this condition, although part of the increased SEP amplitude with aging in BAFME might reflect the effect of physiological aging. This finding was also supported by the significant increase in the myoclonus rating scale with aging in the present study.

A previous SEP study demonstrated that there was no significant relationship between SEP amplitude and age of onset or disease duration of BAFME [15]. However, it was also noted that SEP amplitude tended to increase in aged patients within the same pedigree. Clinically, only the degree of tremulous myoclonus seemed to increase with aging, especially in patients over 70 years of age during a follow-up period of 10 to 15 years $[7,23]$. On the other hand, Striano et al. reported that giant SEPs and enhanced long loop reflex were found in 3 presymptomatic members of a BAFME pedigree and that all 3 members developed tremor and myoclonus several years later [17]. They postulated that the presence of giant SEPs prior to tremor onset might suggest an aberrant integration of somatosensory stimuli that can subsequently involve the motor cortex and cause myoclonus. The present findings and those of Striano et al. suggest an increase in both tremulous myoclonus and SEP amplitude in proportion to aging in patients with BAFME. In other words, cortical hyperexcitability develops at a young age and becomes clinically apparent in adulthood, and then slightly but significantly increases with further aging. This process is so mild that clinical observation may not always be sensitive to delineate the chronological evolution of this condition. Our electrophysiological findings may serve as a biomarker for the pathological mechanism of BAFME. A prospective SEP study along with observation of the clinical course in individual cases of BAFME is warranted to confirm this notion. 
Our findings and those of Striano et al. are also supported by serial observation of clinical and neurophysiological findings in Lafora disease, one of the progressive myoclonus epilepsies [24]. In that report, a gradual increase was shown in SEP amplitude with aging and disease progression, resulting in giant SEPs in Lafora disease. However, there was no significant relationship between the myoclonus rating scale and SEP amplitude in BAFME in our study, which can probably be explained by the relatively small number of examined patients and the simplified myoclonus scale (grading according to 5 ranks from 0 to 4).

In addition, with regard to the terminology of BAFME as it applies at least to Japanese patients, it should be discussed whether both "benign” and "adult” are still appropriate. More appropriate term for Japanese patients would be discussed in the future once similar finding to the present study is confirmed. At the same time, a similarly designated study of European patients could be very important to delineate the similarity between the two apparently endemic syndromes in Japan and Europe. 


\section{Acknowledgement}

This study was supported by the Research Grant (22-3) for the Nervous and Mental

Disorders from the Ministry of Health and Welfare, the Grant-in-Aid for Scientific

Research (C2) 18590935 from the Japan Ministry of Education, Culture, Sports,

Science and Technology (MEXT), and a research grant from the Japan Epilepsy

Research Foundation.

We confirm that we have read the Journal's position on issues involved in ethical publication and affirm that this report is consistent with those guidelines.

\section{Author roles}

Takefumi Hitomi: $\quad$ Research project (Execution), Statistical Analysis (Execution)

Manuscript (Writing of the first draft)

Akio Ikeda:

Research project (Conception and Organization), Manuscript (Review and Critique)

Takayuki Kondo: $\quad$ Research project (Execution)

Morito Inouchi: $\quad$ Research project (Execution)

Riki Matsumoto: $\quad$ Research project (Execution)

Kiyohito Terada: $\quad$ Research project (Execution)

Masutaro Kanda: $\quad$ Research project (Organization)

Masao Matsuhashi: $\quad$ Statistical Analysis (Review and Critique)

Takashi Nagamine: $\quad$ Research project (Organization)

Hiroshi Shibasaki: $\quad$ Research project (Conception and Organization), Manuscript (Review and Critique)

Ryosuke Takahashi: $\quad$ Research project (Organization) 


\section{References}

1. Panayiotopoulos, C.P., Syndromes of idiopathic generalized epilepsies not recognized by the International League Against Epilepsy. Epilepsia, 2005. 46 Suppl 9: p. 57-66.

2. Ikeda, A., et al., Cortical tremor: a variant of cortical reflex myoclonus. Neurology, 1990. 40(10): p. 1561-5.

3. Yasuda, T., Benign Adult Familial myoclonic epilepsy (BAFME). Kawasaki Med J, 1991. 17: p. 1-13.

4. Terada, K., et al., Familial cortical myoclonic tremor as a unique form of cortical reflex myoclonus. Mov Disord, 1997. 12(3): p. 370-7.

5. Okuma, Y., et al., Familial cortical tremor with epilepsy. Parkinsonism Relat Disord, 1997. 3(2): p. 83-7.

6. van Rootselaar, A.F., et al., Familial cortical myoclonic tremor with epilepsy: a single syndromic classification for a group of pedigrees bearing common features. Mov Disord, 2005. 20(6): p. 665-73.

7. Plaster, N.M., et al., Genetic localization of the familial adult myoclonic epilepsy (FAME) gene to chromosome 8q24. Neurology, 1999. 53(6): p. 1180-3.

8. Shibasaki, H., et al., Pathogenesis of giant somatosensory evoked potentials in progressive myoclonic epilepsy. Brain, 1985. 108 ( Pt 1): p. 225-40.

9. Shibasaki, H., et al., Wave form decomposition of 'giant SEP' and its computer model for scalp topography. Electroencephalogr Clin Neurophysiol, 1990. 77(4): p. 286-94. 
10. Ikeda, A., et al., Peri-rolandic and fronto-parietal components of scalp-recorded giant SEPs in cortical myoclonus. Electroencephalogr Clin Neurophysiol, 1995. 96(4): p. 300-9.

11. Luders, H., The effect of aging on the wave form of the somatosensory cortical evoked potential. Electroencephalogr Clin Neurophysiol, 1970. 29(5): p. 450-60.

12. Ikeda, A., et al., Clinical trial of piracetam in patients with myoclonus: nationwide multiinstitution study in Japan. The Myoclonus/Piracetam Study Group. Mov Disord, 1996. 11(6): p. 691-700.

13. Kakigi, R. and H. Shibasaki, Generator mechanisms of giant somatosensory evoked potentials in cortical reflex myoclonus. Brain, 1987. 110 ( Pt 5): p. 1359-73.

14. Tanosaki, M., et al., Effects of aging on central conduction in somatosensory evoked potentials: evaluation of onset versus peak methods. Clin Neurophysiol, 1999. 110(12): p. 2094-103.

15. Kato, M.O., A., A Clinical Study of Benign Adult Familial myoclonus epilepsy. Ann Rep Jpn Epi Res Found, 1999. 11: p. 99-108.

16. Striano, P., et al., A new benign adult familial myoclonic epilepsy (BAFME) pedigree suggesting linkage to chromosome 2p11.1-q12.2. Epilepsia, 2004. 45(2): p. 190-2.

17. Striano, P., et al., Electroclinical and genetic findings in a family with cortical tremor, myoclonus, and epilepsy. Epilepsia, 2005. 46(12): p. 1993-5.

18. Mikami, M., et al., Localization of a gene for benign adult familial myoclonic epilepsy to chromosome 8q23.3-q24.1. Am J Hum Genet, 1999. 65(3): p. 745-51. 
19. de Falco, F.A., et al., Benign adult familial myoclonic epilepsy: genetic heterogeneity and allelism with ADCME. Neurology, 2003. 60(8): p. 1381-5.

20. Depienne, C., et al., Familial cortical myoclonic tremor with epilepsy: the third locus (FCMTE3) maps to 5p. Neurology, 2010. 74(24): p. 2000-3.

21. Ebihara, M.O., H. Meerabux, J. Okubo, Y. Kato, M. Toyota, T. Suto, Y. Yamada, K. Yoshikawa, T., Search for the causative gene in benign adult familial myoclonic epilepsy (BAFME). Ann Rep Jpn Epi Res Found, 2003. 15: p. 57-64.

22. Ikeda, A., S. Kurihara, and H. Shibasaki, Possible anticipation in BAFME: three generations examined in a Japanese family. Mov Disord, 2005. 20(8): p. 1076-7.

23. Uyama, E., Y.H. Fu, and L.J. Ptacek, Familial adult myoclonic epilepsy (FAME). Adv Neurol, 2005. 95: p. 281-8.

24. Orizaola, P. and J. Calleja, [Progressive study of EEG and evoked potentials in Lafora disease]. Rev Neurol, 1998. 27(155): p. 81-5. 
Figure legends

Figure 1: Results of linear regression between age at the time of examination of somatosensory evoked potentials (SEP) and the myoclonus rating scale in 16 patients with benign adult familial myoclonus epilepsy (BAFME).

There is a significant linear regression between age and the myoclonus rating scale in BAFME.

Figure 2: Linear regression between age at the time of SEP examination and N20 (A), P25 (B), and N35 (C) amplitude in 16 patients with BAFME and 19 age-matched control subjects at C3'/C4'.

Bold black line indicates the simple linear regression curve of patients with BAFME and the dotted line indicates that of control subjects. There is a significant linear regression between age and N20 amplitude in both groups, but no significant difference in the regression gradient between the 2 groups was noted (A). A significant linear regression between age and P25 amplitude is evident in both groups, but the regression gradient did not differ between groups (B). In both groups, there is a significant linear regression between age and N35 amplitude. In addition, there is a significant difference between the two groups in the gradient calculated from the linear regression line between N35 amplitude and age at the SEP examination (C). Abbreviations are defined in the legend for Fig.1. 
Figure 3: SEP waveform in representative cases from the same pedigree

Waveforms of SEPs following right median nerve stimulation in an aged patient (Patient 1) (A) and his son (Patient 2) (B). Giant SEPs were found only in Patient 1 (A). The abbreviation SEP is defined in the legend for Fig.1. 


\section{Subjects and methods}

\section{Myoclonus rating scale}

The severity of tremulous myoclonus was classified according to the authors'

previous report as follows: 0: absence of myoclonus, 1: mild myoclonus without disturbance of daily activity, 2: moderate myoclonus with some disturbance in daily activity, 3: severe myoclonus with clear disturbance in daily activity, and 4: marked myoclonus causing incapacity (Table 1) [1]. 


\section{SEP recordings}

SEPs were recorded in a quiet room while the patients and control subjects

were seated in a reclining chair. During the entire recording period, all subjects were kept awake. Electrical stimuli were delivered to the median nerve at the wrist at a fixed rate of $1 \mathrm{~Hz}$ on average. The stimulus intensity was adjusted to produce a clear twitch of the thenar muscle. Scalp electrodes were placed at the contralateral central area; C3 and C4 (C3/C4) in 10 patients (Patients 1, 2, 8-13, 15, 16) and C3' and C4' (C3'/C4') (2 cm posterior to C3/C4) in 6 patients (Patients 3-7, 14) (Table 1). In control subjects, SEPs were recorded both at C3/C4 and C3'/C4'. The electrode impedance was kept below $5 \mathrm{k} \Omega$. The ipsilateral earlobe to the stimulated hand (A1 or A2) was used as the reference in 14 patients and all control subjects, and the linked earlobes (A1 + A2) were used in 2 patients (Patients 3 and 4). The bandpass filter was set to $1-1500 \mathrm{~Hz}$. At least 200 responses were averaged. Two sets of averages were obtained and reproducibility of the SEP waveform was confirmed as shown in Figure 1. We recorded SEPs to bilateral median nerve stimulation in all patients and control subjects except 1 patient (Patient 12) in whom SEPs were recorded to unilateral median nerve stimulation. Thus, we used averaged SEP data for right and left median nerve stimulation in most of the patients and control subjects. 


\section{Results}

\section{Anticonvulsants}

Anticonvulsants (mainly clonazepam and valproic acid) were used for treatment of myoclonus as well as seizures in 9 patients (Patients 2, 5-7 and 11-14 and 16) (Table 1). Anticonvulsants were administered only after a clinical diagnosis was made and SEP recording was done. As a whole, these anticonvulsants including phenytoin were judged to have diminished, though not completely abolished, the myoclonus. The effect of anticonvulsants on seizure was unclear because of its rare occurrence. 


\section{Discussion}

\section{Anticonvulsants}

There was no significant difference in SEP amplitudes related to the use of anticonvulsants in the present patients with BAFME, although SEP amplitudes were reported to decrease after administration of anticonvulsants in patients with BAFME and other cortical reflex myoclonus [2]. Possibly, the negative impact of anticonvulsants on SEP amplitudes as opposed to that shown in the previous study could be explained by the relatively small number of patients in the present study or differences in underlying pathophysiology in BAFME as a possible genetically heterogeneous disorder. 


\section{References}

1. Ikeda, A., et al., Clinical trial of piracetam in patients with myoclonus: nationwide multiinstitution study in Japan. The Myoclonus/Piracetam Study Group. Mov Disord, 1996. 11(6): p. 691-700.

2. Striano, P., et al., Levetiracetam in patients with cortical myoclonus: a clinical and electrophysiological study. Mov Disord, 2005. 20(12): p. 1610-4. 
Table 1 Clinical profile of 16 patients with benign adult familial myoclonus epilepsy

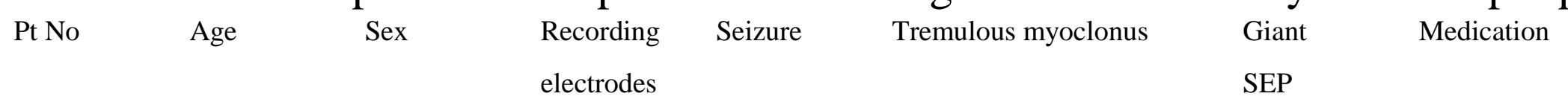

Onset age Onset age Severity\#

\begin{tabular}{|c|c|c|c|c|c|c|c|c|}
\hline $1^{*}$ & 58 & M & C3/C4 & - & 50 & 1 & + & - \\
\hline $2^{*}$ & 34 & M & C3/C4 & 33 & 24 & 1 & - & VPA, CZP \\
\hline 3 & 57 & M & $\mathrm{C}^{\prime} / \mathrm{C}^{\prime}$ & 45 & 40 & 3 & + & - \\
\hline 4 & 75 & M & $\mathrm{C}^{\prime} / \mathrm{C} 4 '$ & 73 & 70 & 3 & - & - \\
\hline 5 & 50 & $\mathrm{~F}$ & $\mathrm{C}^{\prime} / \mathrm{C} 4 '$ & - & 45 & 2 & + & $\mathrm{CZP}$ \\
\hline 6 & 31 & $\mathrm{~F}$ & $\mathrm{C}^{\prime} / \mathrm{C} 4^{\prime}$ & 17 & - & 0 & + & VPA \\
\hline 7 & 35 & M & $\mathrm{C}^{\prime} / \mathrm{C} 4 '$ & 30 & 10 & 2 & + & VPA \\
\hline 8 & 27 & F & C3/C4 & - & 24 & 2 & - & - \\
\hline 9 & 36 & F & C3/C4 & - & 33 & 2 & + & - \\
\hline $10^{+}$ & 38 & M & C3/C4 & 30 & 35 & 2 & + & - \\
\hline $11^{+}$ & 74 & $\mathrm{~F}$ & C3/C4 & - & 40 & 4 & + & VPA, CZP \\
\hline 12 & 69 & M & C3/C4 & 35 & 25 & 2 & + & PHT, CZP, DZP \\
\hline 13 & 76 & $\mathrm{~F}$ & C3/C4 & 62 & 59 & 3 & + & VPA \\
\hline $14 \&$ & 40 & $\mathrm{~F}$ & $\mathrm{C}^{\prime} / \mathrm{C}^{\prime}$ & 28 & 20 & 1 & + & CZP, CBZ, PB, TPM \\
\hline $15 \&$ & 72 & $\mathrm{~F}$ & C3/C4 & 62 & 50 & 2 & + & - \\
\hline 16 & 37 & $\mathrm{~F}$ & C3/C4 & 23 & - & 0 & + & VPA \\
\hline
\end{tabular}

* *, + , and $\&$ indicate the same pedigree

- \# severity indicates: 0: absence of myoclonus, 1: mild myoclonus without disturbance of daily activity,

2: moderate myoclonus with some disturbance of daily activity, 3: severe myoclonus with clear disturbance of daily activity

4: marked myoclonus causing incapacity (Ikeda et al., 1996)

- Giant SEP: P25 > $10.0 \mu \mathrm{V}$ or N35 > $8.1 \mu \mathrm{V}$ for younger group (50 years or less) and P25 > $20.0 \mu \mathrm{V}$ or N35 > $14.8 \mu \mathrm{V}$ for older group (more than 50 years of age).

- VPA: valproic acid, CZP: clonazepam, PHT: phenytoin, DZP: diazepam, CBZ: carbamazepine, PB: phenobarbital, TPM: topiramate 


\section{Table 2 Results of SEP study in BAFME patients and control subjects}

$$
\begin{array}{ll}
\text { Patients with BAFME } & \text { Control subjects } \\
(\mathrm{N}=16, \text { age: } 51 \pm 18 \text { years }) & (\mathrm{N}=19 \text {, age: } 49 \pm 18 \text { years })
\end{array}
$$

Latency (ms)

$\begin{array}{lll}\text { N20 } & 18.2 \pm 1.5 & 18.5 \pm 0.9 \\ \text { P25 } & 23.0 \pm 1.6 & 24.6 \pm 2.4 \\ \text { N35 } & 31.6 \pm 4.0 & 30.0 \pm 2.0\end{array}$

Amplitude $(\mu \mathrm{V})$

N20

$$
\begin{aligned}
& 2.8 \pm 1.2^{*} \\
& 11.4 \pm 6.1^{*} \\
& 19.2 \pm 11.5^{*}
\end{aligned}
$$$$
4.3 \pm 1.8
$$

P25

$6.7 \pm 3.4$

N35

$3.7 \pm 2.9$

$\cdot P<0.05$ as compared with control subjects at C3'/C4' by the Mann-Whitney U test. 
Figure 1

Myoclonus rating scale

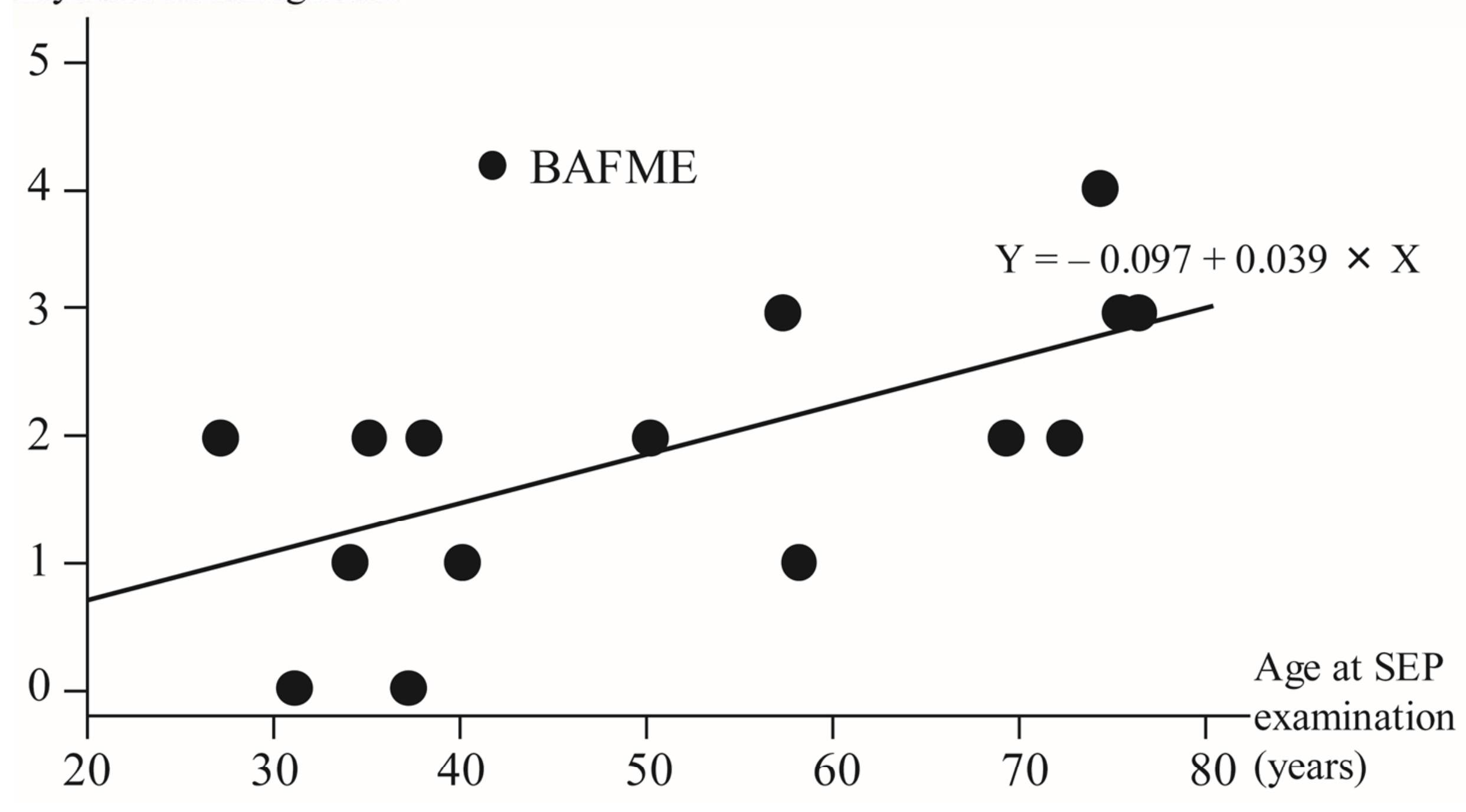


Figure 2

A:
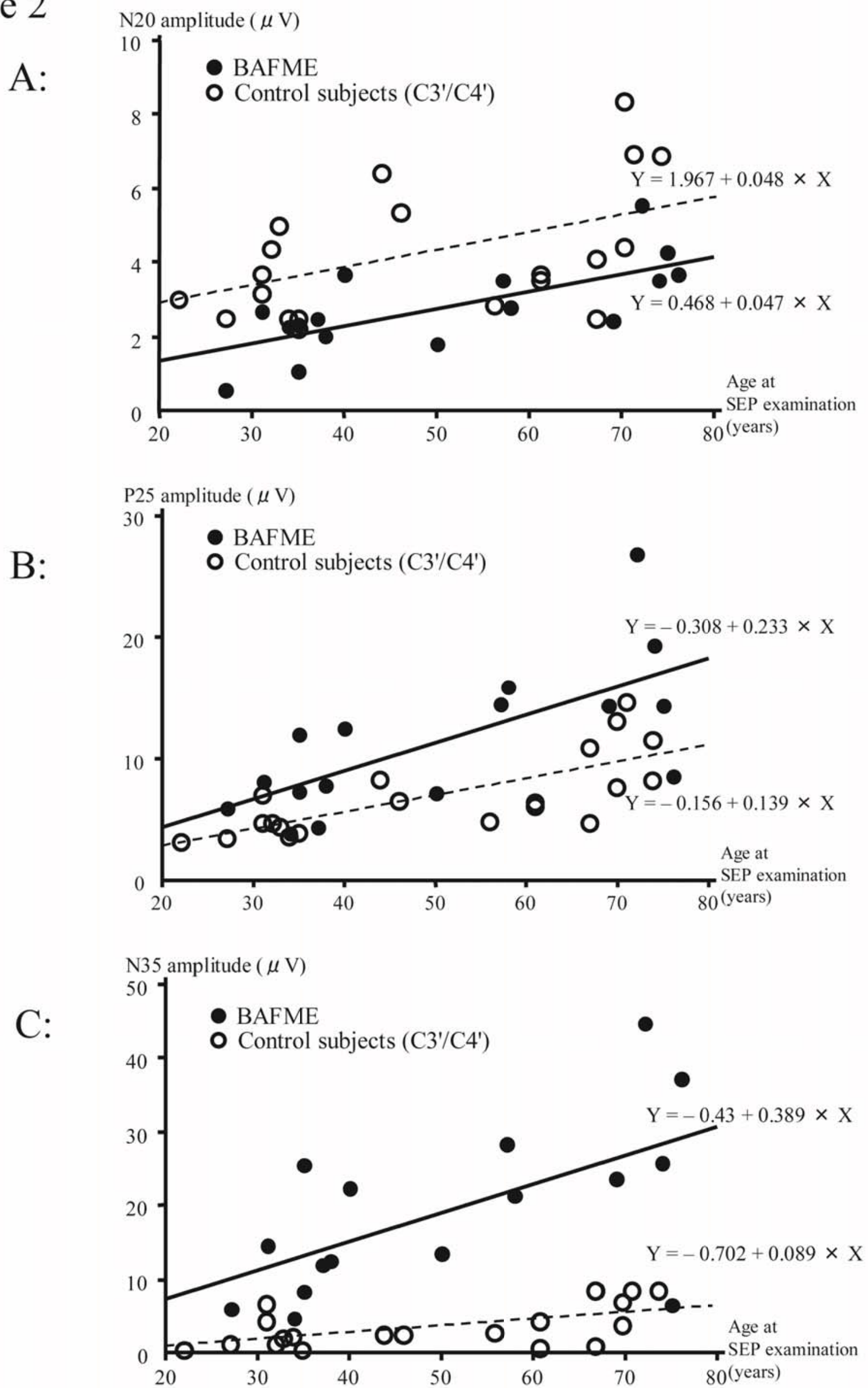
Figure 3

A: Patient 1 (58-year-old, male)

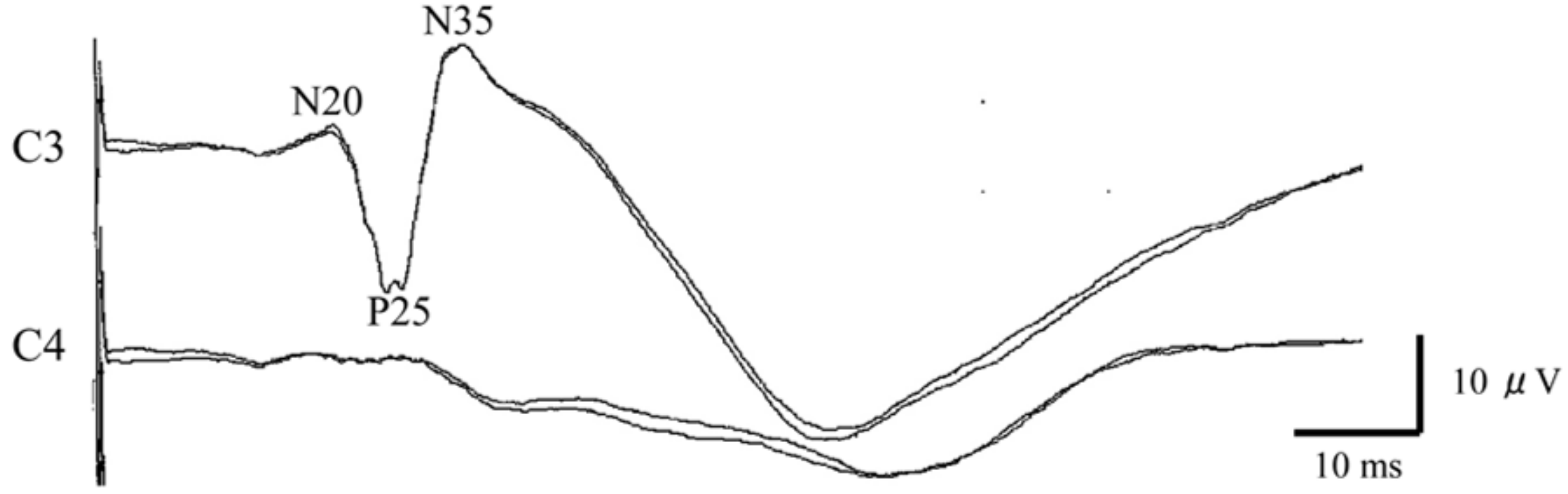

B: Patient 2 (34-year-old, male, son of Patient 1)

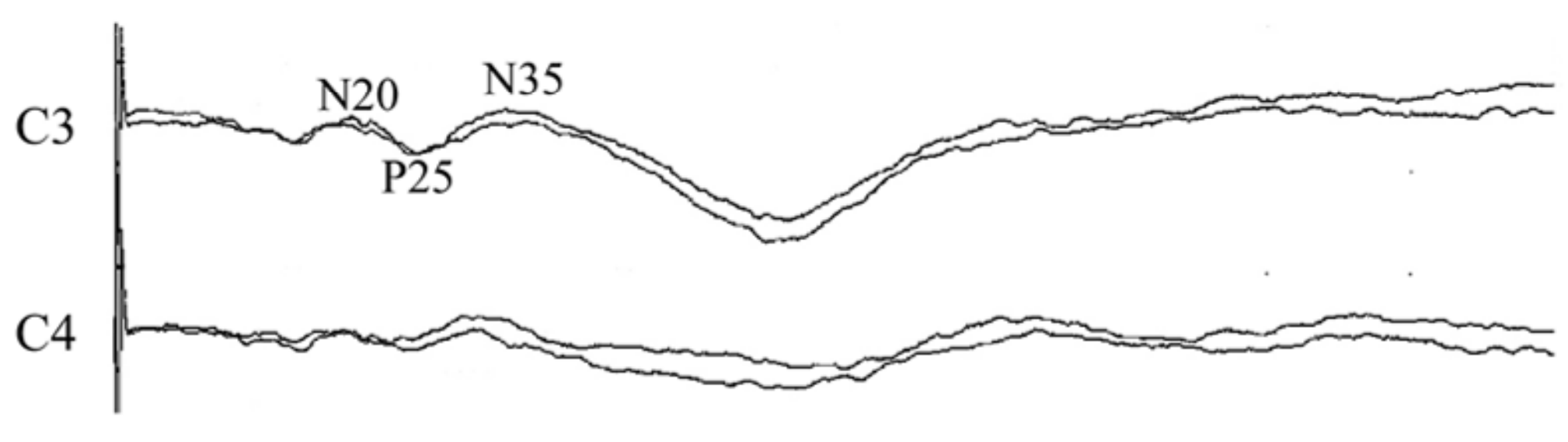

